\title{
A Fine Tune Neural Network for Smart Home Automation System Using Internet of Things (IoT)
}

\author{
O. E. Taylor ${ }^{1}$, P. S. Ezekiel ${ }^{2}$ \\ Department of Computer Science, Rivers State University, Port Harcourt, Nigeria ${ }^{1,2}$
}

\begin{abstract}
A coincide of technologies in Artificial Intelligence and inescapable computing as well as the development of powerful sensors and actuators has gotten interest in the development of smart mediums to transpire and uphold important functions in Daily Living Activities (ADLs). This system proposes an intelligent system for home automation using Internet of things. First, we propose a model using fine tune neural network algorithms. This fine tune neural network has to do with transfer learning. By transfer learning, we mean transferring a knowledge of an existing model into the new model for faster training and for better training performance. This transfer learning was implemented in python by importing mobileNetv2 from keras.applications using tensorflow framework. Secondly, we proposed an emotion expression dataset from which our proposed model will learn to recognize the emotion of a person in the home by means of facial recognition. The model will be able to tell how the person feels, if the person is happy, sad, angry and so on. From the person's emotional expression, the model will be able to automate any home appliances according to how the person feels. The emotional dataset used in our work is the FER 2013 dataset, which was downloaded from kaggle.com. After successfully training, we had an accuracy of about $88 \%$ on all the classes, and had an accuracy of $97 \%$ on two of the image classes. Our trained model was saved and exported to web using python flask, were we carried out our testing on a live we camera video.
\end{abstract}

Keywords: Fine tune Neural Network, Home Automation, Internet of Things, Home Appliances

\section{INTRODUCTION}

A coincide of technologies in Artificial Intelligence and inescapable computing as well as the development of powerful sensors and actuators has gotten interest in the development of smart mediums to transpire and uphold important functions in Daily Living Activities (ADLs). The need for such advancement in technologies to be built is underlined by populace maturing, the expense of formal medical care, and the significance people put on leftover free in their own homes. People should have the option to finish day-by-day living exercises like eating, dressing, cooking, drinking, perusing, taking medication, resting, to work freely at home. Computerizing action acknowledgment is an urgent advance towards observing a shrewd home inhabitant's useful wellbeing and assisting them with playing out these activities successfully [1]. Smart Home Systems are the sub-group of regular computing which incorporates smart innovation for giving solace, wellbeing, security and energy decrease. At the point when this application is constrained by machine knowledge to give condition mindful settings, benefits and encourage controller, it fundamentally improves client comfort. Further expansion of computerized machine control and availability administrations can improve the personal satisfaction also. Internet of things (IoT) that contain various sensors can recognize temperature, light, stable, distance, pneumatic force, movement which go about as various purposes of information sources. Since there is an immense measure of information included Machine learning can be applied to the current Home computerization systems to cause it to perform incredibly all around based off the client's feelings [2].

A smart home is a home that gives its inhabitants the solace, comfort and simplicity of activity of gadgets consistently, independent of where the occupant really is inside the house. A smart home normally comprises of electrical apparatuses like lighting, fans, forced air systems, room-radiators, air-coolers and microwave and so on; and electronic contraptions like TV, PCs, sound systems, PCs, music-frameworks and cell phones and so forth. Every one of these machines and devices can be associated and controlled distantly, over a protected channel utilizing Wi-Fi or web through programming application, from the inside or outside the house. A smart home comprises of three components: (I) inner home organization, (ii) clever control and (iii) home computerization with wired/remote access passages. These frameworks and gadgets inside a home can likewise be overseen either from inside home, or can be connected to administrations and frameworks from outside the home [3]. These machines and devices are for the most part associated with explicit sensors, to make these naturally adjusted to specific circumstances and thusly cause the inhabitants to feel good. A versatile smart home would be the one that uses Artificial Intelligence procedures to find designs in the occupants in every day exercises, and create robotization rules and activities that mirror these activities [4]. When these frameworks become mindful of 


\section{International Journal of Advanced Research in Computer and Communication Engineering}

Vol. 10, Issue 2, February 2021

DOI 10.17148/IJARCCE.2021.10204

the prerequisites of occupants, the inhabitants would then be able to be given a superior living encounter, by foreseeing their future necessities and perform routine errands. The point is to diminish actual developments and work by the people, by detecting and proactively reacting to their requirements. This significant application space is anticipated to consistently increment later on [5].

This paper presents a fine tune neural network for smart home automation system using internet of things. In other to make the home to be smarter, we will be using a computer vision to detection the emotion of a person in the house via facial recognition on a live video surveillance camera.

\section{RELATED WORKS}

An Intelligent, Secure, and Smart Home Automation System [6] present a creative idea of a smart home that utilizes an Artificial Intelligence algorithm (Support Vector Machine) for smart dynamic and furthermore utilizes blockchain innovation to guarantee ID and confirmation of the IoT gadgets. Arising blockchain innovation assumes an essential part by giving a dependable, secure, and decentralized instrument for recognizable proof and validation of the IoT gadgets utilized in the proposed home robotization framework. Besides, the SVM classifier is applied to group the situation with gadgets utilized in the proposed smart home mechanization framework into one of the two classifications, i.e., "ON" and "OFF."/is framework depends on Raspberry Pi, $5 \mathrm{~V}$ hand-off circuit, and a few sensors. A versatile application is created utilizing the Android stage. Raspberry Pi going about as the worker keeps up the data set of every apparatus. /e HTTP web interface and apache worker are utilized for correspondence among the Android application and Raspberry Pi. /e proposed thought is tried in the lab and genuine to approve its adequacy and helpfulness. It is additionally guaranteed that the equipment and innovation utilized in the proposed thought are modest, effectively accessible, and replicable. /e trial results feature its importance and approve the confirmation of the idea.

HEMS-IoT: A Big Data and Machine Learning-Based Smart Home System for Energy Saving [7] presents HEMS-IoT, a major information and Artificial Intelligent based smart home energy supervision system for home solace, security, and energy saving. they utilized the J48 Artificial Intelligent algorithm and Weka API to learn client practices and energy utilization designs and group houses regarding energy utilization. In like manner, they depended on RuleML and Apache Mahout to create energy-saving suggestions dependent on client inclinations to protect savvy home solace and security. To approve our framework, they present a contextual investigation where we screen a brilliant home to guarantee solace and security and decrease energy utilization.

Smart Home Automation using IoT and Deep Learning [1] centers on the idea of home robotization by utilizing their cell phone information to perceive the occupants ' human exercises. In their paper, they propose a smart home framework that perceives human exercises through a Long Short Term Memory (LSTM) Deep Learning calculation and afterward performs foreordained errands dependent on the perceived movement. They fabricated a LSTM model that can anticipate human movement from 200 time-step succession with more than 97\% accuracy on the test set.

Smart Home Automation Using Machine Learning Algorithms [8] utilize ideas of various Machine Learning algorithms alongside computer vision to shape together a smart learning robotized framework that controls lighting, sound and different gadgets dependent on the client's feeling. They talk about the guideline and strategies in which a smart home robotization framework utilizing Machine Learning is being actualized. Their proposed framework is associated with the current Home Wireless Network. Various Sensors and gadgets are interfaced with the info/yield ports on the Raspberry Pi (Single Board Computer). Their framework likewise has an Apache controlled Web-worker that fuses the User interface for any gadget on the organization to distantly trigger some other machines in the home. These solicitations are sent by regular Hypertext Transfer Protocol (http) to the Raspberry Pi. Different solicitations from the User to the sensors/gadgets that were utilized are recorded in a data set so they fill in as the information that can be utilized by the worker running profound learning calculations. After effectively preparing of their calculations they had an exactness of about $66.6 \%$ and $70.80 \%$ for both $\mathrm{CNN}$ and Linear support vector machine on all 7 classes.

A Voice-Controlled Multi-Functional Smart Home Automation System [9] proposes a multi-utilitarian smart Home Automation System (SHAS), where clients can utilize voice-orders to control their home-machines and devices, for various functionalities and purposes. The proposed framework can be adjusted to a client's voice and perceive the voiceorders, autonomous of the speaker's very own qualities like emphasize. The framework is expected to be savvy, adaptable and powerful. The voice order acknowledgment is accomplished utilizing a devoted equipment module and an Arduino miniature regulator board for orders preparing and control. Execution assessment is done by building up a multi-useful scaled down model of the SHAS. Consequences of the tests directed are very encouraging. The model SHAS can be utilized for changing over existing homes into brilliant homes at moderately ease and with accommodation.

Early Detection System for Gas Leakage and Fire in Smart Home Using Machine Learning [10] proposed a method of coordinating gas spillage and fire frameworks inside savvy home climate to upgrade security utilizing minimal effort and less-energy utilization gadgets through M2M standard correspondence conventions. They proposed a compelling dataflow framework for social event helpful data to an essential issue in M2M home organization. We conveyed our framework model with sensors and perform tests under various conditions. From their informational collection, we 


\title{
International Journal of Advanced Research in Computer and Communication Engineering
}

\author{
Vol. 10, Issue 2, February 2021
}

DOI 10.17148/IJARCCE.2021.10204

applied a regulated AI measure on a few calculations for noticing occasions which are not adjust to the normal example, and anticipating the degree of dangers of risk. Their framework is likewise ready to send a notice to alarm pertinent people and continue appropriately.

IoT based Smart Home Automation System using Sensor Node [11] made use of the sensor and identified the presence or nonappearance of a human item in the housework appropriately. Their proposed method additionally gives information about the energy devoured by the house proprietor consistently as message. Likewise, it checks, the degree of gas in the gas chamber in the event that it arrives at lesser than the edge, it consequently books the gas and sends a reference number as a message to the house proprietor. The proposed system is sent and tried for different conditions. At last, in their work, the working model of our proposed arrangement is created as a model and clarified as a working model.

SLASH: Self-Learning and Adaptive Smart Home Framework by Integrating IoT with Big Data Analytics [12] presented an overview such most recent technologies that can prompt another worldview of a intelligent home, in view of grouping the fundamental parts of such frameworks and present the past work in every segment. Also, SLASH system is proposed for planning and executing intelligent home frameworks that are both versatile and self-learning. Their structure recommends incorporating IoT across each home with an enormous organization associated with Big Data analyser. Such a motor that supports dissecting occupants' practices for an enormous scope can empower another kind of home computerization that relies upon machine learning and creates on-going robotization choice over the long run. This methodology holds a few difficulties that are considered all through the structure or state for future upgrade.

Comfy Smart Home using IoT [13] discussed about how Internet of Things is utilized to associate different gadgets and how it very well may be actualized. Further it portrays the working of face acknowledgment framework utilizing Artificial intelligent and how it very well may be inserted in security locks. They examined about centralization of the whole framework utilizing portable application distantly and close by. It will likewise be illuminating how the current innovations can be made open for disabled individuals. At long last, it will be giving a short thought regarding how different gadgets and advances can be casted on a solitary stage without the client adapting up to numerous applications, making everything epitomized in a solitary application. The primary innovations that are utilized to construct brought together home mechanization framework is Internet of Things for availability, Machine Learning to make the framework intelligent and progressed and an application through which the framework can be controlled whether it is an android based portable application or Web based application. The significant errand of such a framework is to concentrate the whole framework to make it really programmed.

\section{MeTHODOLOGY}

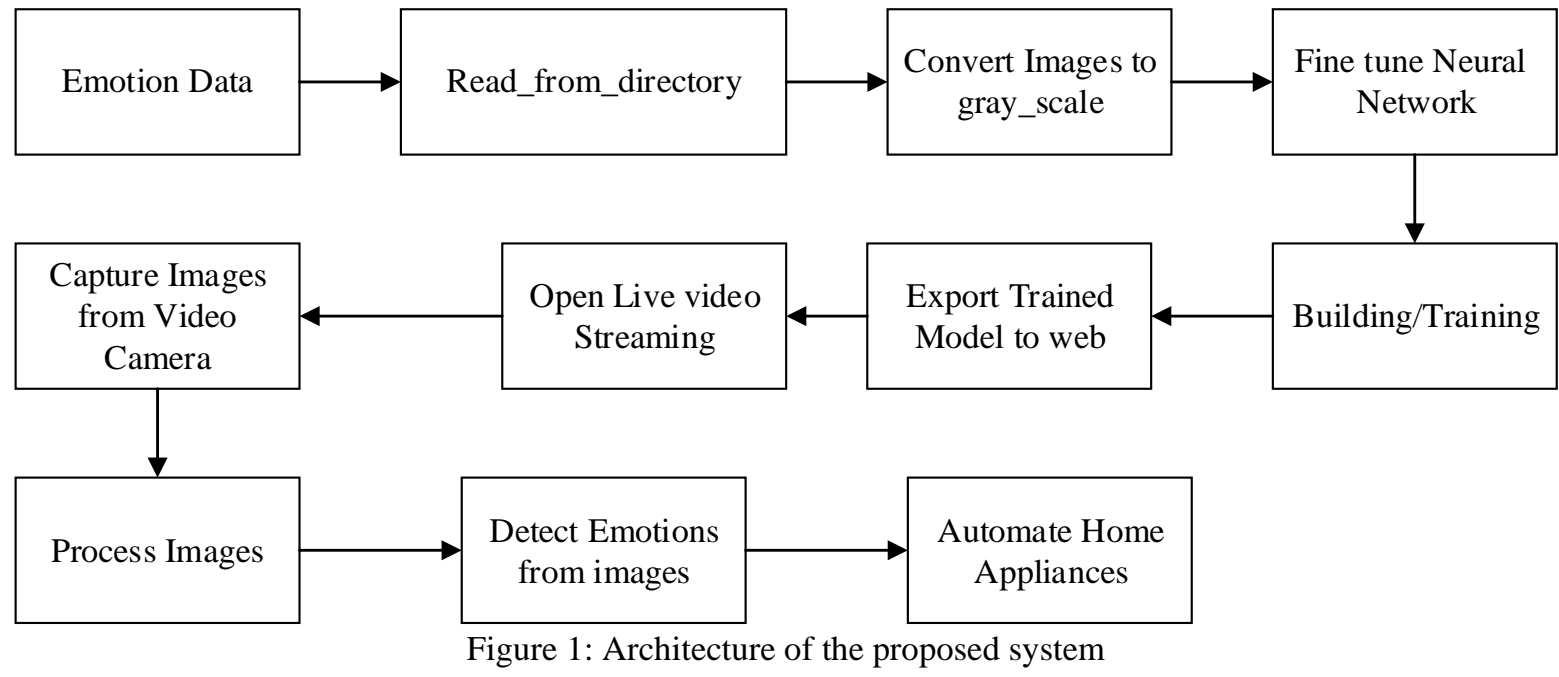

Emotion Dataset: The proposed system uses FER 2013 dataset, which was downloaded from kaggle.com. The dataset contains about seven classes of human emotions all in image format. The seven classes are angry, disgust, fear, happy, sad, surprise and neutral. Each of the class contains images not less than 500, the dataset has a total image of about 35 , 473. The following are the problems of the dataset.

$\begin{aligned} \text { i. } & \text { Imbalance Problem } \\ \text { ii. } & \text { Intra-Class Variation } \\ \text { iii. } & \text { Occlusion } \\ \text { iv. } & \text { Contrast Variation } \\ \text { v. } & \text { Eyeglasses } \\ \text { vi. } & \text { Outliers }\end{aligned}$




\section{International Journal of Advanced Research in Computer and Communication Engineering}

Vol. 10, Issue 2, February 2021

\section{DOI 10.17148/IJARCCE.2021.10204}

The solutions to the above problems are Data augmentation and avoiding overfitting.

Read_From Directory: The image dataset was been read from directory by calling cv2.imshow() function. The function will contain the directory folder and path. The cv2 was been imported from the opencv library. The oepncv library is a used for computer vision.

Convert_images_to_gray_scale: This has to do with the conversion of the images to gray scale by calling the cv2.cvtColor function, which then takes the image name and the colour name that you want to pass the image to. It can be gray scale or a normal image colour.

Fine Tune Neural Network: This has to do with a transfer learning, by transfer learning we mean integrating information from an existing model into the new model architecture so as to make training time fast and efficient. Figure 2 shows the architecture of a fine tune neural network which has to do with transfer learning.

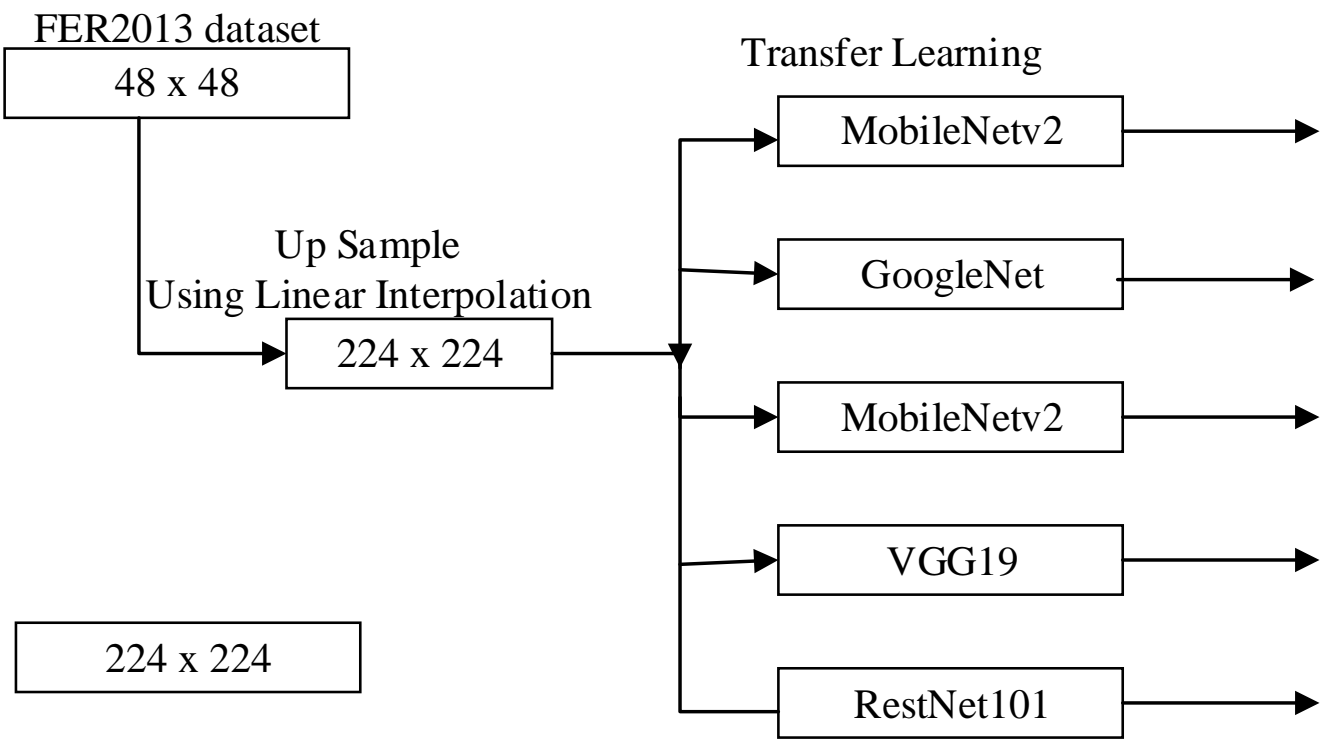

Figure 2: Architecture of a fine tune neural network (Transfer Learning)

Building/Training model: The model will be built by loading a pre-trained model from tensorflow.keras. applications.MobileNetv2(). Here the pre-trained model has different convolutional layer type and output shape. So we will be selecting the one that best suit our proposed model. So we selected the one with an output shape of seven. We will then set our learning rate to be 0.001 , optimizer to be RSMprop and loss to be categorical_conssentropy, epoch to be 128 .

Exporting trained model: The trained model will be saved and exported to web using python flask. Flask is a micro framework for web applications. Here we will be using html,css and JavaScript for frontend design and python for the backend.

Opening live video streaming/ capturing images from video: we will be starting a live video by calling cv2.videoCapture(), which will contain 0 for local web camera and rtsp://username:password@ip_address:554/user=username_password='password'_channel=channel_number_stream=0. sdp' for cctv camera. The video will be read by calling the read() function. CascadeClassifier will be used to detect multiple images from the video camera.

Process Images/Detect Emotions from images: The image processing has to do with the resizing of the image to the appropriate scale value as same as the trained model, so as to obtain a better classification result. After processing, the images will be passed to the trained model to classify what class that the images fall under.

Automate Home: Here, the system will be using the emotion detected from the facial expression images to decide what to do at home. E.g, either to turn off/on bulbs, turn the fan and television on and off.

\section{IV.RESULT AND DISCUSSION}

This system proposes an intelligent system for home automation using Internet of things. First, we propose a model using fine tune neural network algorithms. This fine tune neural network has to do with transfer learning. By transfer learning, we mean transferring a knowledge of an existing model into the new model for faster training and for better training performance. This transfer learning was implemented in python by importing mobileNetv2 from keras.applications using tensorflow framework. Secondly, we proposed an emotion expression dataset from which our proposed model will learn 


\section{International Journal of Advanced Research in Computer and Communication Engineering}

Vol. 10, Issue 2, February 2021

\section{DOI 10.17148/IJARCCE.2021.10204}

to recognize the emotion of a person in the home by means of facial recognition. The model will be able to tell how the person feels, if the person is happy, sad, angry and so on. From the person's emotional expression, the model will be able to automate any home appliances according to how the person feels. The emotional dataset used in our work is the FER 2013 dataset, which was downloaded from kaggle.com. The dataset contains about seven classes of human emotions all in image format. The seven classes are angry (4593), disgust (547), fear (5121), happy (8989), sad (6077), surprise (4002) and neutral (6198). The dataset contains a total image of 35, 473 images. The dataset has some imbalance problem, which we resolved by means of augmentation. By augmentation of data, we mean increasing the amount of data by adding modified copies of an existing data. By this, the problem of overfitting during training of the model is solved. After solving the above stated problems of the dataset, our newly modified dataset was read into memory by (1) declaring the memory path. (2) by setting the path to be os. path.join(directory). (3) by reading the directory path using cv2.imshow(os.path.join(path, img), where cv2 is a module in opencv library and os is being imported from the os library. After successfully setting and reading of images from directory, we resized the images into 224 x 224 pixel, and then, we passed it to the pre-trained model with an input_shape $=((224,224), 3$,$) , batch_size of 128, learning_rate of 0.0.1. After$ successfully training, we had an accuracy of about $88 \%$ on all the classes, and had an accuracy of $97 \%$ on two of the image classes. Our trained model was saved and exported to web using python flask. Here we created a mini web page that will load a live web camera, and this camera will detect a human face, once it detects a human face, it will recognize the emotional expression of the human through the knowledge of the trained model. Once it's recognizes the emotional expression of the human, them it automatically automates home appliances depending on the emotional expression it recognized from the human face. This automation can be (1) Turning on/off the light bulb (2) turning on/off fan (3) turning on/off the television sets. Figure 3-5 shows some images presents in anger, happy and Neutral class. Figure 6 shows the training process of the proposed model. Figure 7 shows the accuracy of the proposed model for each epoch level. Figure 8 shows the loss values during the training process. In figure 9 the system detected the human face as been neutral in the study room, automatically the system automates the light in the study room. Table 1 shows comparison result between our system and other existing system.

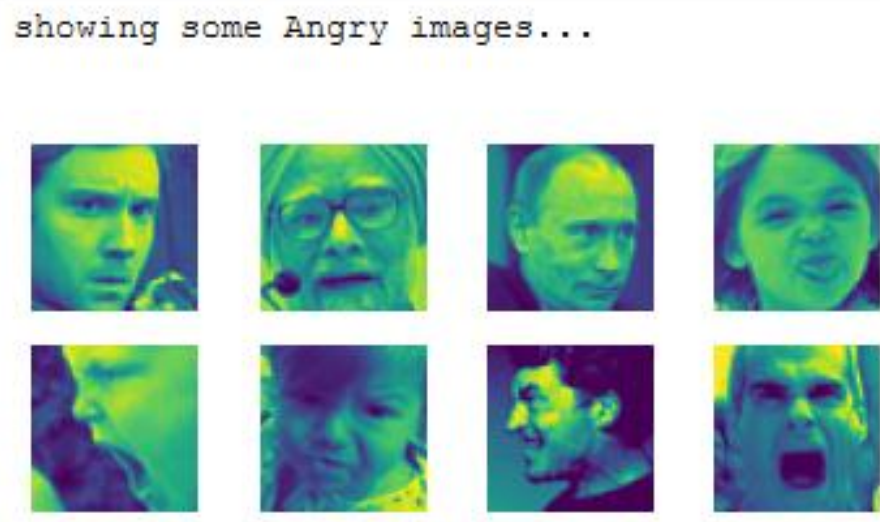

Figure 3: Showing some Angry Images

showing some Happy images...
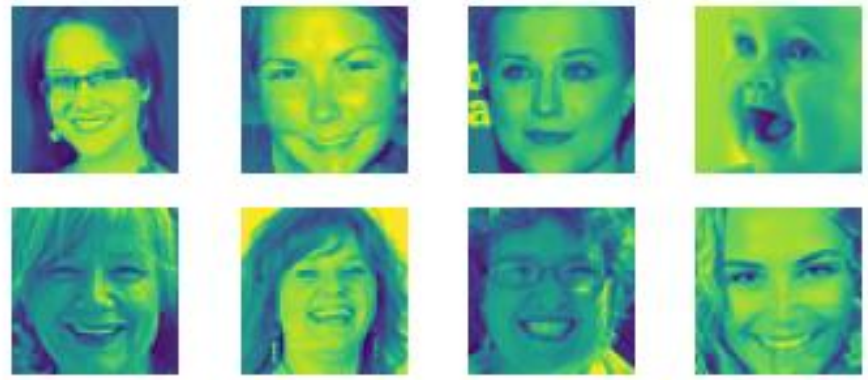

Figure 4: showing some Happy Images 


\section{International Journal of Advanced Research in Computer and Communication Engineering}

Vol. 10, Issue 2, February 2021

DOI 10.17148/IJARCCE.2021.10204

showing Neutal images...
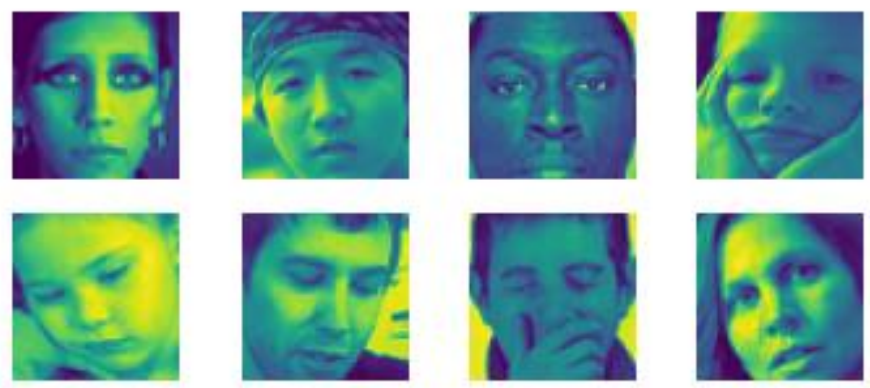

Figure 5: showing some Neutral Images

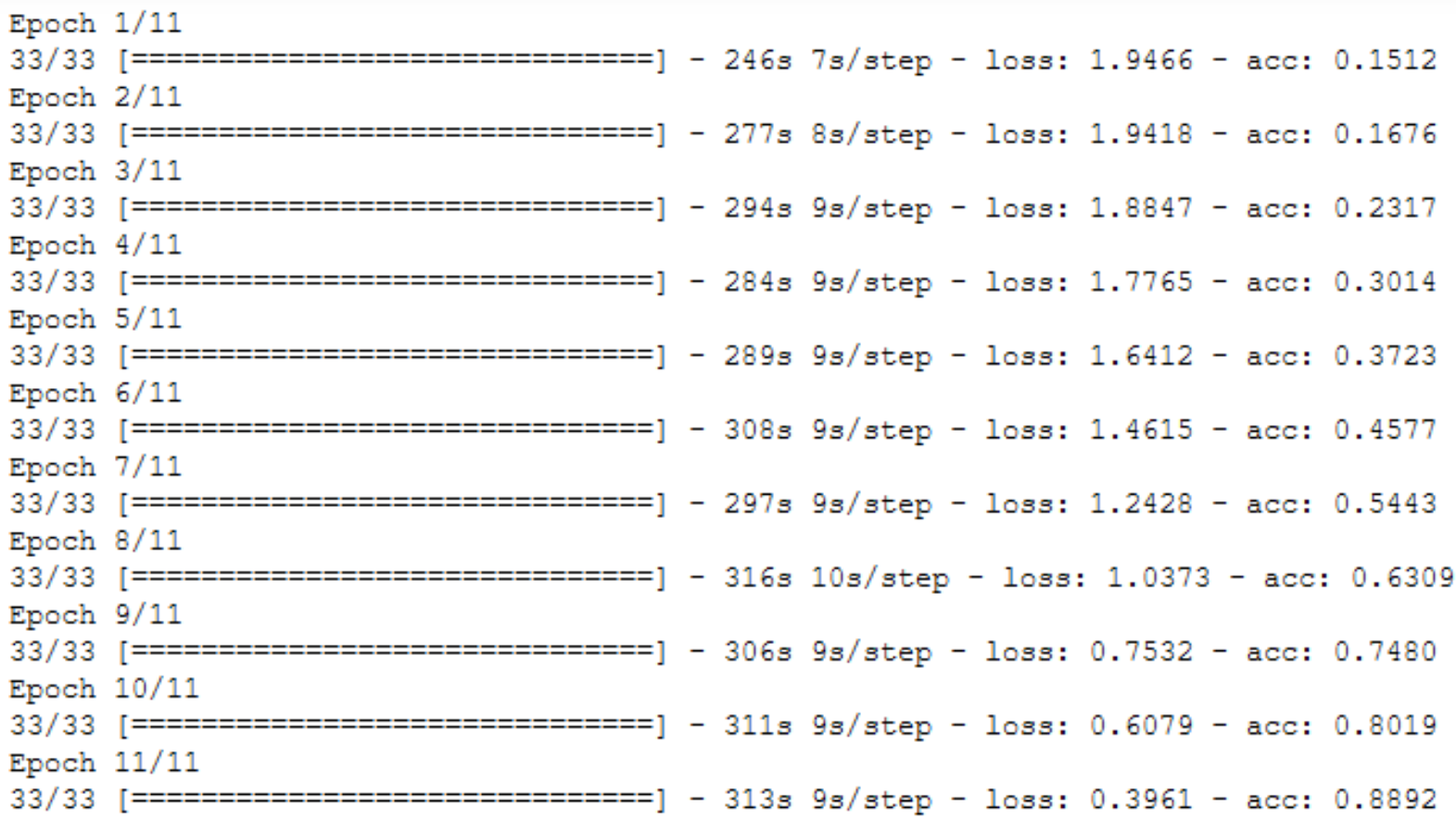

Figure 6: showing training process of the proposed model 
International Journal of Advanced Research in Computer and Communication Engineering

Vol. 10, Issue 2, February 2021

DOI 10.17148/IJARCCE.2021.10204

\section{Training accuracy with epochs}

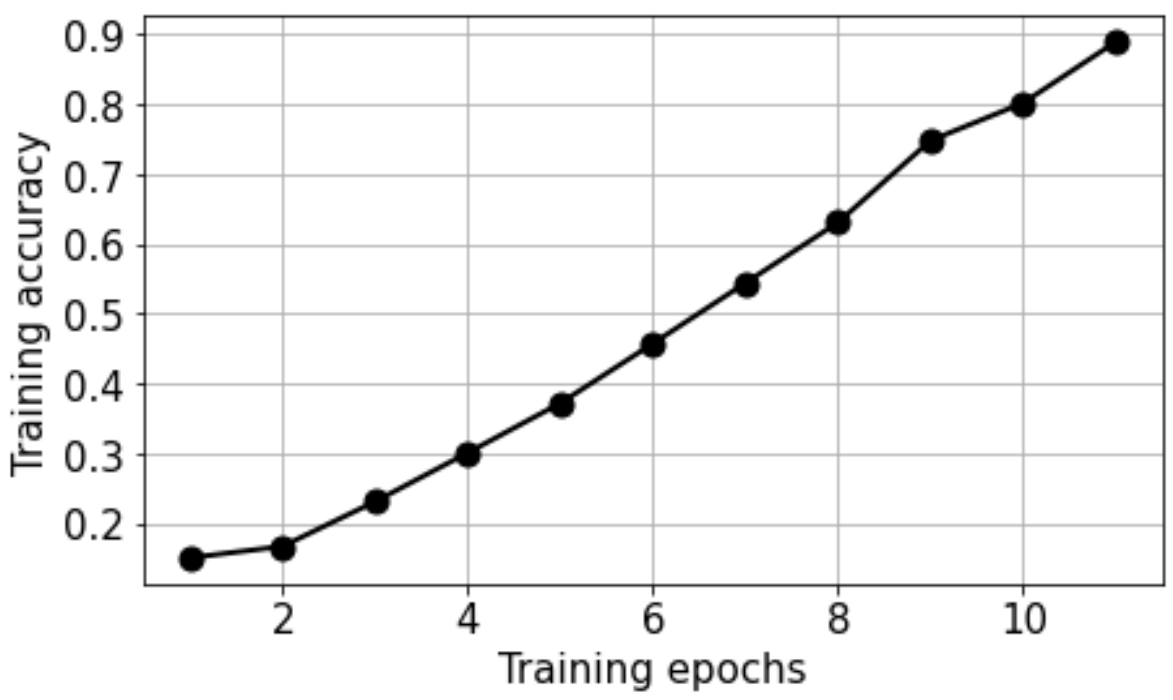

Figure 7 showing accuracy vs training epoch

Training loss with epochs

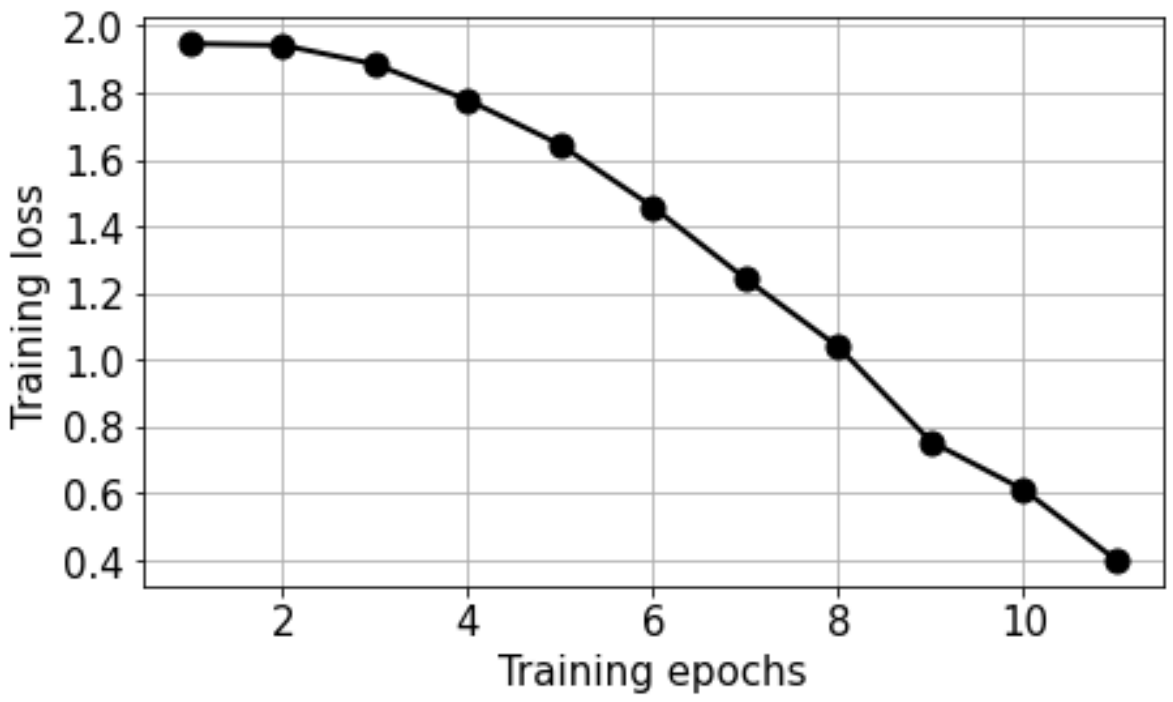

Figure 8: showing the loss values during the training process 


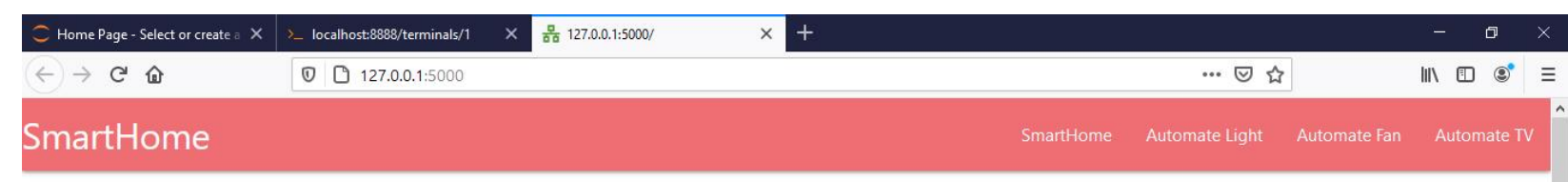

\section{Home Automation System by means of emotional expression}
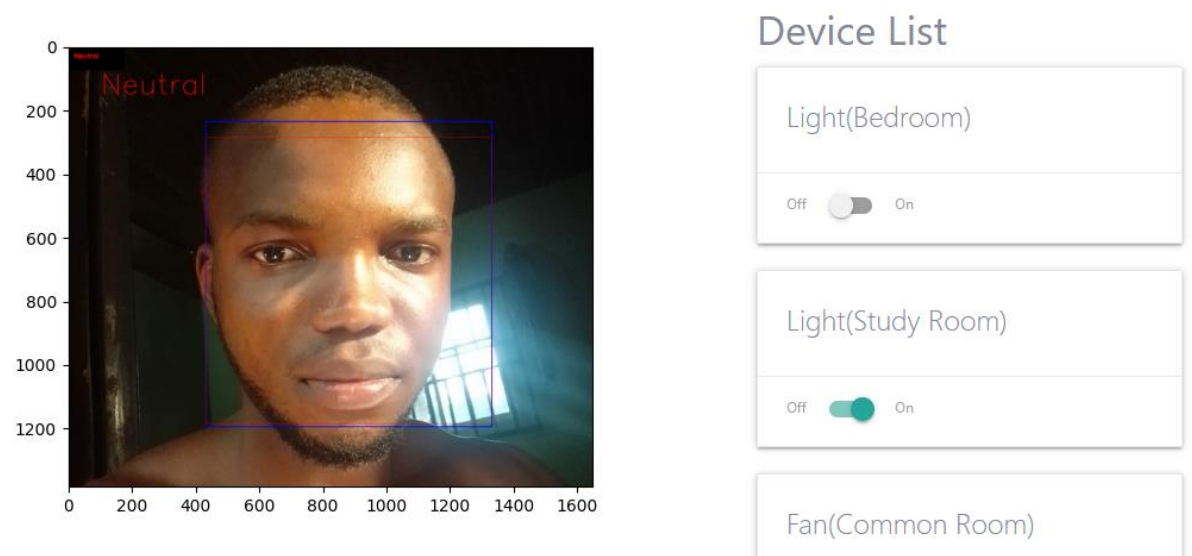

0 Type here to search

节

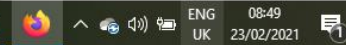

Figure 9: The system detected the human face to be neutral, and automatically it automates the bulb in the study room by turning it on.

Table 1: System Comparison with other Existing System

\begin{tabular}{|c|c|c|c|}
\hline No. & Arthur & Title & Accuracy \\
\hline 1 & Jaiha et.al, 2020 & $\begin{array}{c}\text { Smart Home Automation Using Machine Learning } \\
\text { Algorithms }\end{array}$ & $66.6 \%$ \\
\hline 2 & Our model & $\begin{array}{c}\text { A Fine Tune Neural Network for Smart Home } \\
\text { Automation system using Internet of Things (IoT) }\end{array}$ & $88.89 \%$ \\
\hline
\end{tabular}

Table 1 shows the comparison with our work with other existing system. Here, we both proposed a system that Automate home appliances by detecting the emotion of a person using facial recognition. We both use the same algorithm but on a different emotional expression dataset. Here they trained their model having an accuracy of about $66.6 \%$ while we trained our model having an accuracy of about $88.9 \%$. The table above shows that our proposed system is better than the existing system in terms of accuracy.

\section{CONCLUSION AND FUTURE WORK}

Technology has been increasing rapidly; many manual processes are being eliminated on daily basics, and are being taken online. A smart or an intelligent home is a home that all appliances have an online remote access in turning the home devices on/off. In this paper, we proposed an intelligent system that will learn the emotional expression of a person in the human, and automatically automate the appliances of the devices in the homes accordingly to the emotional expression of the human in the home through the help of Internet of things (IoT). The emotional expression can either be angry, happy, sad, disgust, neutral and fearful. If the system detects the human emotion to be angry, it automatically turns off the whole appliances of the home. This work can further be extended by using a live CCTV camera that will be mounted over the house. This CCTV camera will be linked to the system using an IP address. By this, it will cover the whole appliances in the home individually.

\section{REFERENCES}

[1] R. D. Manu, S. Kumar, S. Snehashish, K.S. Rekha "Smart Home Automation using IoT and Deep Learning" International Research Journal of Engineering and Technology (IRJET) 6(4), pp. 1-4, 2019.

[2] M. R. Alam, M. B. I. Reaz and M. A. M. Ali, "A review of smart homes: Past, present, and future." IEEE Transactions on Systems, Man, and Cybernetics, Part C (Applications and Reviews) 42(6), pp. 1190-1203, 2012.

[3] D.-Y. L. Li Jiang and B. Yang, "Smart Home Research,” in Proc. International Conference on Machine Learning and Cybernetics, 2004, vol. 2, Aug 2004, pp. 659-663. 


\section{International Journal of Advanced Research in Computer and Communication Engineering}

Vol. 10, Issue 2, February 2021

\section{DOI 10.17148/IJARCCE.2021.10204}

[4] M. Chan, D. Esteve, C. Escriba, and E. Campo, “A Review of Smart `homes-Present State and Future Challenges,” Comput. Methods Prog. Biomed., vol. 91, no. 1, pp. 55-81, Jul 2008.

[5] M. R. Kamarudin and M. A. F. M. Yusof, "Low Cost Smart Home Automation via Microsoft Speech Recognition," International Journals of Engineering and Sciences, vol. 13, no. 3, 2013

[6] R. Majeed, N. A. Abdullah, I. Ashraf, Y. B. Zikria, M. F. Mushtaq, M. Umer “An Intelligent, Secure, and Smart Home Automation System”, Scientific Programming: Towards a Smart World 2020, pp.1-14. 2020.

[7] I. Machorro-Cano, G. Alor-Hernández, M. A. Paredes-Valverde, L. Rodríguez-Mazahua, J. L. Sánchez-Cervantes, J. O. Olmedo-Aguirre "HEMSIoT: A Big Data and Machine Learning-Based Smart Home System for Energy Saving”, Energies 13(15), pp.2-24, 2020.

[8] H. Jaihar, N. Lingayat, P. Sapan, Vijaybhai, G. Venkatesh, K. P. Upla "Smart Home Automation Using Machine Learning Algorithms" 2020 International Conference for Emerging Technology (INCET) Belgaum, India pp.5-7, 2020.

[9] Y. Mittal, P. Toshniwal, S. Sharma, D. Singhal, R. Gupta \& V. K. Mittal "A Voice-Controlled Multi-Functional Smart Home Automation System", Annual IEEE Indian Conference (INDICON), pp.1-6, 2015.

[10] L. Salhi, T. Silverston, T. Yamazaki,T. Miyoshi "Early Detection System for Gas Leakage and Fire in Smart Home Using Machine Learning" IEEE International Conference on Consumer Electronics (ICCE), pp.1-6, 2019.

[11] H. Singh, V. Pallagani, V. Khandelwal, U. Venkanna “IoT based Smart Home Automation System using Sensor Node", 4th Int'1 Conf. on Recent Advances in Information Technology | RAIT-2018 |

[12] M. Sultan, K.N. Ahmed "SLASH: Self-Learning and Adaptive Smart Home Framework by Integrating IoT with Big Data Analytics" Computing Conference, London, UK., pp.1-10 2017.

[13] S. Jadon, A. Choudhary1, H. Saini, U. Dua , N. Sharma , I. Kaushik "Comfy Smart Home using IoT" International Conference on Innovative Computing and Communication (ICICC-2020). 